\title{
BPM - BUSINESS PROCESS MANAGEMENT COMO PRÁTICA DE GESTÃO EM UMA EMPRESA METALÚRGICA COM ESTRATÉGIA DE PRODUÇÃO ETO - ENGINEER-TO-ORDER
}

\section{BPM - BUSINESS PROCESS MANAGEMENT AS PRACTICE MANAGEMENT IN A METALURGIC COMPANY WITH ETO - ENGINEER-TO-ORDER PRODUCTION STRATEGY}

\author{
Gabriel Sperandio Milan ${ }^{1}$; Francis André Soso ${ }^{2}$ \\ ${ }^{1}$ Universidade de Caxias do Sul - UCS - Caxias do Sul - RS - Brasil \\ gsmilan@ucs.br \\ ${ }^{2}$ Universidade de Caxias do Sul - UCS - Caxias do Sul - RS - Brasil \\ soso.francis@gmail.com
}

\begin{abstract}
Resumo
$O$ artigo apresenta uma metodologia de gestão de processos baseado no ciclo de vida de gerenciamento de processos proposto pela ABPMP - Association of Business Process Management Professionals, bem como as atividades, técnicas e práticas de gestão que habilitam a realização do BPM - Business Process Management, compreendendo as fases de planejamento e estratégia, análise de processo, desenho e modelagem de processo, implementação de processo, monitoramento e controle de processo e refinamento de processos. De forma complementar, foram apresentadas as ferramentas que habilitam e suportam o ciclo de vida do gerenciamento de processos. Para tanto, foi proposta a utilização de um roteiro para aplicação da metodologia em uma empresa metalúrgica no sentido de aumentar a eficiência do seu ciclo de pedidos. Com tal intervenção proposta, espera-se que haja uma maior conexão entre a estratégia de atendimento à demanda adotada pela empresa (ETO - Engineer-to-Order) e o gerenciamento de seu processo produtivo, resultando em melhor atendimento aos clientes .
\end{abstract}

Palavras-chave: BPM - Business Process Management, gestão de processos, estratégia de produção.

\section{Introdução}

Em ambientes de negócios competitivos, as organizações executam atividades interdependentes, relacionando recursos humanos, produtivos, tecnológicos e financeiros de forma a maximizar seu desempenho. O sucesso nos negócios, portanto, passa pela compreensão e pelo gerenciamento da relação entre as atividades exercidas na organização (FNQ, 2010).

O conjunto das atividades realizadas em uma sequência lógica e com o objetivo de produzir algo que tenha valor para um cliente denomina-se processo (HAMMER; CHAMPY, 1994). Para 
Smith e Fingar (2003), os processos são a fonte do diferencial competitivo de uma empresa. É a forma pela qual uma organização entrega valor aos seus clientes. Consoante isso, a gestão de processos busca a otimização sistêmica deste conjunto de atividades, que é possível de ser realizada através da prática do BPM (Business Process Management - Gestão de Processo de Negócio), que envolve a descoberta, o projeto e a entrega de processos de negócios (BALDAM; VALLE; PEREIRA, 2008; MANOILOV; DELIISKA, 2008). Inclusive, a melhoria dos processos de negócio diminui o esforço cognitivo dos colaboradores de uma empresa na solução de problemas aumentando a sua performance (BALASUBRAMANIAM et al., 2005; NEAL, 2008) e, por consequência disso, o desempenho do negócio (JESTON, 2008), principalmente pelo alinhamento do gerenciamento de processos com os objetivos e as estratégias corporativas (NEAL, 2008; HUANG; MEI, 2003).

Segundo Davenport (1994), o foco da visão de negócio não deve ser mais por função ou por departamento (área), e sim por processos-chave, o que é corroborado por Hammer (2001), que afirma que empresas orientadas por processos representam o melhor modelo de gestão para um mundo em constante mudança. $\mathrm{O}$ gerenciamento destes processos cria práticas organizacionais mais fortes, que conduzem a processos mais eficazes, o que gera retorno mais elevado para as partes interessadas (ABPMP, 2009).

Uma das formas de gerenciar os processos de negócio é por meio da prática do ciclo de vida do BPM, que pode ser sumarizado por um conjunto gradual e interativo de atividades que incluem: planejamento, análise, desenho e modelagem, implantação, monitoramento e controle e refinamento dos processos de negócio (ABPMP, 2009). Este ciclo de vida é contínuo e compreende as atividades gerenciais exercidas para garantir a entrega de valor ao cliente de forma eficiente.

É oportuno ressaltar que o BPM é fortemente suportado pelo uso extensivo de Tecnologia de Informação (TI), devido à sua complexidade e abrangência. Seu uso permite que os processos sejam monitorados e evoluídos com a velocidade que o mercado exige (CRUZ, 2008). E a implementação de tecnologias e de sistemas de informação envolve mais do que simplesmente aspectos funcionais (WOLTER; MEINEL, 2010; SLACK; LEWIS, 2005). Para o sucesso da implementação do BPM, suportado pela TI (Tecnologia da Informação), é essencial que haja o apoio da alta direção e principalmente o alinhamento dos processos com a estratégia organizacional (CRUZ, 2008).

A verificação da aplicabilidade de um modelo de Gestão de Processos de Negócio, assim como os fatores críticos para o sucesso do projeto e as ferramentas de TI necessárias se dará a partir do estudo da viabilidade de implantação do BPM em uma indústria metalúrgica localizada na Serra Gaúcha, a qual tem sua estratégia de produção orientada ao pedido do cliente (ETO - Engineer-toOrder - Projetar sob Encomenda). 


\section{Referencial teórico}

\subsection{BPM - Business Process Management}

A administração de uma empresa em tempos de rápidas mudanças exige uma estrutura dinâmica e flexível, capaz de gerar valor para todos stakeholders simultaneamente, utilizando os recursos humanos, tecnológicos, financeiros e produtivos disponíveis de forma eficiente e criativa, buscando eliminar por completo os desperdícios do negócio (HAMMER; CHAMPY, 1994; JESTON, 2008). Neste contexto, a gestão de processos busca entregar resultado que tenha valor para o cliente, na forma de produtos e serviços, organizando uma série de atividades transacionais da organização (SMITH; FINGAR, 2003; HAMMER, 20001; RUMMLER; BRACHE, 1994). As atividades que fazem parte de um processo recebem uma entrada (input), adicionam valor (processo de transformação) a ela e entregam uma saída (output) ao cliente, estando diretamente relacionadas à coordenação do fluxo dos objetos na organização (PAIM; CARDOSO; CAULLIRAUX, 2009; GONÇALVES, 2000a).

Uma das formas de adicionar valor a organização é torná-la fácil, amigável, para que o cliente faça negócios com a empresa, e isso é possível através da ordenação lógica das atividades de trabalho no tempo (HAMMER, 2001). Esta ordenação lógico-temporal dos meios necessários para atingir os objetivos serve de guia para empresa durante o percurso e forma sua estrutura para a ação, possibilitando o alcance das metas (DAVENPORT, 1994; CRUZ, 2008; GONÇALVES, 2000b).

A gestão de processos de negócio é viável a partir da identificação e organização das atividades necessárias para produzir e entregar bens e serviços que atendam as expectativas do cliente. A noção de processos de negócio elimina a visão e a administração departamental do negócio, ou seja, gerencia os processos ponta-a-ponta, ou finalísticos, que são o conjunto relevante de atividades que cruzam os limites funcionais da empresa para, ao final, entregar valor ao cliente (ABPMP, 2009). De acordo com Baldam, Valle e Pereira (2008), a gestão de processos de negócio - BPM envolve a descoberta, projeto e entrega de processos de negócio. O gerenciamento envolve um conjunto de técnicas e ferramentas que permitam identificar, desenhar, executar, documentar, medir e monitorar processos de negócio para que entreguem os resultados pretendidos e reforcem a estratégia organizacional, tornando a organização eficiente e eficaz por completo (ABPMP, 2009; MANOILOV; DELIISKA, 2008).

Ainda segundo esses autores, os fatores críticos determinantes para o sucesso da implantação do BPM em uma organização são: apoio da alta direção, alinhamento dos processos com a estratégia do negócio, capacitação das pessoas e a gestão da mudança. A adoção de uma 
metodologia que oriente todo o trabalho e a condução do projeto de implementação por pessoas experientes aumentam a chance de sucesso.

Uma forma de organizar o processo de implementação do BPM é por meio da divisão do projeto em duas fases distintas, a modelagem do estado atual $(A S I S)$ e a modelagem e otimização no estado futuro $(T O B E)$. A primeira fase representa o registro e entendimento dos processos na situação atual através da criação de um modelo. A segunda fase, por sua vez, consiste em concentrar os esforços da equipe para um refinamento dos processos atuais. Este refinamento deve objetivar reduzir os desperdícios de custo, tempo, erros, etc., e deve ser orientado por modelos de referência e pela adoção das melhores práticas do mercado (PAIM; CARDOSO; CAULLIRAUX, 2009; BALDAM; VALLE; PEREIRA, 2008).

As soluções para melhoria dos processos visam obter mais resultado utilizando menos recursos, e estão diretamente associadas ao nível de maturidade que os processos possuem, podendo ser desde inicial, repetível, definido, gerenciado até o otimizado. Frequentemente estas melhorias são viabilizadas por ações relacionadas a pessoas, tecnologias, processos, cultura e desempenho, entre outros, e uma das formas de compreender e organizar as ações e fatores relacionados à gestão destes processos de negócio é sumarizada no ciclo de vida BPM (ABPMP, 2009; BALDAM; VALLE; PEREIRA, 2008; OLIVEIRA, 2009).

\subsection{0 ciclo de vida BPM}

O ciclo de vida proposto pela ABPMP (2009) representa a prática gerencial da gestão de processos de negócio realizada de forma contínua e inclui as atividades de: planejamento; análise; desenho e modelagem; implantação; monitoramento e controle; e refinamento, sendo habilitadas, suportadas e restringidas ao longo do tempo por fatores como culta, valores e crenças da organização. Este modelo é uma ferramenta extremamente útil para orientar a implantação do BPM em uma organização principalmente por ser um modelo simples e intuitivo.

A utilização de um modelo estruturado para gestão do ciclo de vida dos processos de negócio é importante para criar na organização a consciência necessária para a evolução da visão departamental para a visão de processos, desenvolvendo atividades que atendam aos clientes e a organização de forma racional, gerando valor para o negócio (JESTON; NELIS, 2006; RUMMLER; BRACHE, 1994; HARRINGTON, 1991).

Segundo a ABPMP (2009), as fases do ciclo de vida BPM podem ser resumidas da seguinte forma: (i) Planejamento e Estratégia: o ciclo de vida BPM começa com a elaboração de um plano, definindo objetivos e metas e estratégias para a geração de valor para o cliente; (ii) Análise: sua finalidade é entender os processos atuais da organização; (iii) Desenho e Modelagem: representar como o trabalho ponta-a-ponta ocorre de modo a entregar valor aos clientes, criando um 
entendimento e uma compreensão do negócio e deve avaliar todos os fatores favoráveis e desfavoráveis ao processo; (iv) Implementação: é a fase de transformação do processo atual para o processo proposto; (v) Monitoramento e Medição (controle): a contínua medição e monitoramento fornecem informações para o gestor tomar decisões de ajuste, afim de atingir os objetivos dos processos; e (vi) Refinamento: a realização de ajustes no curso de ação proporciona a melhoria incremental necessária para o processo evoluir continuamente.

\subsection{Estratégia de produção}

Uma vez adotada uma estratégia corporativa, que viabilize a realização da missão do negócio, e estabelecido os objetivos diretivos a serem alcançados, é importante tomar decisões coerentes nas funções empresariais, tipicamente representadas pelas área de Marketing, Produção (ou Operações), Recursos Humanos e Finanças, que apóiem e suportem a realização da estratégia principal do negócio. Estas funções empresariais são hierarquicamente subordinadas às diretrizes estabelecidas no nível superior e, portanto, devem interagir para contribuir para o alcance dos objetivos corporativos (WHEELWRIGTH, 1994; PORTER, 2005).

A estratégia de produção é essencial para entender a forma como uma empresa industrial se posiciona no mercado para responder à demanda, e é relevante para o contexto do estudo, pois a opção da empresa de projetar produtos sob encomenda é determinante para a análise do tempo, custo e complexidade do seu ciclo de pedidos (KLIPPEL; ANTUNES; PELLEGRIN, 2005). O objetivo centar da estratégia de produção é garantir que os processos de produção e entrega de valor aos clientes estejam alinhados com a intenção estratégica da empresa (CORRÊA; CORRÊA, 2006).

O objetivo da estratégia de produção é apoiar, implementar e impulsionar a estratégia do negócio, organizando recursos e processos para criar e manter competências adequadas para entregar produtos e serviços com valor ao cliente. Este papel é determinante para o alcance dos objetivos organizacionais e é possível a partir da adoção de políticas, planos, comportamentos e ações intrinsecas à função produtiva (CORRÊA; CORREA, 2006; SLACK et al., 1999; WHEELWRIGTH, 1984).

Nesta direção, Slack et al. (1999) destacam que a função produção busca cinco diferentes objetivos de desempenho para garantir uma vantagem de longo prazo para a organização, são eles: qualidade, rapidez, confiabilidade de entrega, flexibilidade e custo adequado. Estes objetivos de desempenho ainda são classificados em critérios qualificadores, aqueles que habilitam a empresa a disputar um pedido e os critérios ganhadores de pedido, que representam um diferencial para o negócio, e prioritariamente a organização deve buscar desenvolver os critérios e objetivos que o consumidor mais valoriza. Rosemann, Recker e Flender (2008), por sua vez, enfatizam a relevância estratégica das empresas possibilitarem uma maior flexibilidade em seus processos produtivos, ou 
seja, elaborar a capacidade intrínseca a tais processo em responderem às demandas do ambiente competitivo.

A estratégia de produzir contra um pedido, mais especificamente a iniciativa de projetar um produto sob encomenta (ETO - engineer-to-order) está relacionada ao tipo de produto a ser fabricado pela empresa, focada em produtos customizados para o cliente. Este tipo de estratégia de produção requer maior flexibilidade da organização e tende a torná-la menos eficiente e, consequentemente, tornando o produto geralmente mais caro que em uma produção para estoque (CHENG, 2006; DAVIS; AQUILANO; CHASE, 2001; HICKS; MCGOVERN, 2009).

De acordo com Corrêa, Gianesi e Caon (2008), a característica determinante da estratégia de produção de projetar sob encomenda ETO é que o projeto, a manufatura e a montagem final dos produtos são executadas somente a partir da solicitação do cliente. Mesmo com esta incerteza a opção de criar estoques estratégicos de matéria-prima é recomendada para praticantes desta estratégia de produção, pois protege o ambiente produtivo de oscilações do mercado fornecedor e permite a entrega dos produtos dentro do prazo acordado. Ao sincronizar as atividades produtivas ao processo de desenvolvimento de produtos (PDP), operações baseadas na estratégia ETO podem incrementar o ciclo de desenvolvimento de produtos (CHENG, 2006; FORTIN; HUET, 2007; HICKS; MCGOVERN, 2009).

A opção de projetar e produzir sob encomenda proporciona à organização condições de oferecer ao mercado produtos customizados, soluções únicas, realizadas com base na necessidade e capacidade financeira do cliente e também nas condições técnicas e operacionais da organização. Em contrapartida, a opção de projetar sob encomenda aumenta significativamente o ciclo do pedido e a entrega final, aumentando, também, os riscos financeiros e operacionais a que a empresa está submetida, assim como a incerteza quanto ao processo produtivo e relação comercial, devendo esta estratégia ser exaustivamente planejada e eficientemente controlada pelo praticante (CHENG, 2006; TRAPPEY et al., 2009).

\subsection{Tecnologia da Informação (TI) habilitadora do BPM}

A gestão de processos de negócio, BPM, busca criar um entendimento e uma linguagem comum entre profissionais com visão de negócio e profissionais com visão de TI. A prática do BPM é fortemente habilitada e suportada pelo uso de TI, permitindo o uso eficiente dos métodos de gestão dos processos ponta-a-ponta, aumentando substancialmente o desempenho das organizações (PAIM; CARDOSO; CAULLIRAUX, 2009; ABPMP, 2009; DE SORDI, 2005; HAMMER; CHAMPY, 1994; SLACK; LEWIS, 2005).

Para Cruz (2008), o BPM é um conjunto de métodos e tecnologias que integram logicamente e cronologicamente fornecedores, a organização e seus clientes, e este conjunto de tecnologias da 
informação que operacionalizam as metodologias são chamados de BPMS - Business Process Management System. Sua função é automatizar e controlar os processos por meio de execução de regras de negócio, liberando o trabalhador para a execução da análise das situações cotidianas, e não a realização das operações burras e repetitivas. As tecnologias associadas ao BPM são maduras suficientemente para prover o esteio necessário à automação dos processos e das atividades essenciais, provendo um nível de segurança mínimo às operações (TRACY, 2007).

A organização e o gerenciamento dos processos de negócio, por meio dos BPMS, permite aos gestores que os processos definidos e descritos sejam, de fato, implementados na prática. Além disso, deveriam suportar, de forma transparente, múltiplas instâncias de um dado processo ou de uma determinada tarefa (MANOILOV; DELIISKA, 2008). Os softwares BPMs são tidos com a evolução dos sistemas de workflow (controle de fluxo) e utilizam a mesma lógica destes sistemas, que é baseada no controle de roles, rules e routes (papéis, regras e rotas), sendo capaz de executar o controle e direcionamento do tráfego de informações na organização. Esta integração entre sistemas de informação e atividades finais dos processos tornam a execução das atividades de negócio mais eficientes, eficazes e efetivos, possibilitando a geração de valor para o cliente (SLACK; LEWIS, 2005; BALDAM; VALLE; PEREIRA, 2008; CRUZ, 2008; MANOILOV; DELIISKA, 2008; PAIM; CARDOSO; CAULLIRAUX, 2009).

\section{Metodologia de pesquisa}

\subsection{Caracterização do ambiente em estudo}

O estudo foi desenvolvido em uma indústria metalúrgica localizada na Serra Gaúcha, uma empresda fabricante de equipamentos industriais e fornecedora de soluções customizadas para seus clientes. A empresa possui 48 anos de solidez e tradição e é líder nacional no segmento em que atua e também participante do mercado internacional. Sua busca pela excelência é representada, entre outras certificações e prêmios de inovação, pela certificação ISO 9001 que possui desde 1998.

A empresa tem como principal valor disponibilizar tecnologias inovadoras para seus clientes, desenvolvendo parcerias duradouras e atuando de forma responsável e rentável. A busca pelo reconhecimento como referência em seu segmento, o uso intensivo de TI para automação dos processos e o alto grau de customização dos equipamentos que fornece torna seu processo de atendimento de pedido bastante especializado, dispendioso e complexo.

Devido à natureza de sua estratégia de produção de projetar seus produtos orientados pelo pedido do cliente (ETO - engineer-to-order) e da relação desta estratégia com o ciclo do pedido, este processo será o foco da pesquisa. A organização possui uma estrutura para atendimento ao cliente composta por quatro áreas distintas, porém interligadas, que abrigam quinze diferentes 
funções exercidas por uma equipe de quarenta colaboradores ordenados de forma a suprir as necessidades de especificações técnicas e conhecimentos de mercado para atender aos clientes.

\subsection{Objetivos do trabalho}

O objetivo geral deste estudo é propor a implementação do BPM habilitado e suportado pelo uso de TI como prática de gestão para aumentar a eficiência do atendimento ao cliente em uma indústria metalúrgica localizada na Serra Gaúcha praticante da estratégia de produção ETO.

Em acréscimo, foram definidos os seguintes objetivos específicos: (i) apresentar as estratégias para implementação do ciclo de vida BPM proposto pela ABPMP; (ii) definir características necessárias às ferramentas de TI para habilitar e suportar o BPM; (iii) demonstrar a situação atual do ciclo de pedidos; e (iv) analisar as mudanças projetadas a partir da implementação do BPM e das ferramentas de TI na empresa.

\subsection{Técnicas e procedimentos de pesquisa adotados}

A pesquisa científica tem como objetivo básico a busca por informações que possam auxiliar na solução de um problema, empregando técnicas científicas que garantem o rigor do método e proporcionam o aprimoramento do processo de produção de conhecimento. Esta investigação deve ser viável de ser executada pelo pesquisador e possuir um sentido, uma finalidade, para que tenha utilidade para a sociedade (GIL, 1999). A pesquisa científica é um conjunto de procedimentos sistemáticos, baseados no raciocínio lógico, que tem por objetivo encontrar soluções para os problemas propostos mediante o emprego de métodos científicos (ANDRADE, 2006). Pesquisar significa propor uma investigação e procurar respostas para esta busca (SILVA; MENEZES, 2001).

A pesquisa realizada teve como objetivo ampliar o conhecimento sobre o tema proposto e por isso teve caráter exploratório e abordagem qualitativa. A fonte de dados empíricos para o estudo é uma indústria metalúrgica da Serra Gaúcha, fabricante de equipamentos customizados para seus clientes e orientada por uma estratégia de produção ETO.

Segundo Godoy (1995), a pesquisa qualitativa tem como características o caráter descritivo, o enfoque indutivo na análise, tem o ambiente como fonte direta de dados e o pesquisador como instrumento preocupado com o processo e com o resultado da pesquisa. Ainda para Godoy (1995), a confiabilidade e a validade da pesquisa qualitativa é de difícil determinação, dada a natureza subjetiva da pesquisa e dos resultados que são situacionais e limitados ao contexto.

Os procedimentos técnicos adotados na pesquisa foram à pesquisa bibliográfica do tema proposto junto a teses, artigos científicos, dissertações e livros e também a técnica da observação direta assistemática dos fatos junto ao campo de estudos. Segundo Silva e Menezes (2001), a 
pesquisa bibliográfica utiliza material já publicado para iniciar e contribui para aprofundar o conhecimento do problema, assim como descobrir o que já foi explorado sobre o tema. Em um primeiro momento foi realizada uma pesquisa conceitual sobre BPM, incluindo o ciclo de vida BPM proposto pela ABPMP e as tecnologias habilitadoras e de suporte a sua implementação. Após pesquisou-se sobre estratégia produtiva para estabelecer a relação entre uma estratégia de produção e o gerenciamento do ciclo do pedido, conforme a realidade verificada na empresa em estudo.

A pesquisa assistemática ou observação não estruturada é a técnica de observação direta e intensiva que se caracteriza por examinar o cotidiano de forma livre para depois realizar a análise dos fenômenos observados (HENNINK; HUTTER; BAILEY, 2011; REMLER; VAN RYZIN, 2011). Esta observação foi realizada em campo, observou a rotina de toda equipe de atendimento ao cliente da empresa onde o pesquisador foi somente observador não participante. Vale comentar que a finalidade desta pesquisa aplicada não é esgotar a discussão sobre o tema proposto, mas buscar uma solução para o problema da organização pesquisada envolvendo verdades e interesses locais (GIL, 1999; HENNINK; HUTTER; BAILEY, 2011), mas verificar a aplicabilidade das ferramentas de gestão de processo propostas pela ABPMP (2009), o que permite projetar os ganhos advindos da adoção do modelo de gestão proposto.

\section{Desenvolvimento do estudo}

\subsection{Técnicas gerenciais relacionadas à gestão de processos}

O desenvolvimento do trabalho investigou os conceitos, técnicas e métodos disponíveis na literatura nacional e internacional, assim como cases de sucesso na adoção desta prática gerencial por diversas empresas pequenas, médias e grandes, em vários segmentos de mercado. A finalidade de verificar os aspectos envolvidos na implementação do BPM é aumentar significativamente a chance de sucesso na implementação na empresa pesquisada e alcançar os objetivos propostos na pesquisa. Antes da apresentação das estratégias necessárias para que o projeto atinja seus objetivos faz-se necessário algumas considerações. Os fatores críticos para o sucesso do projeto de implementação dependem fortemente do alinhamento da estratégia da organização com suas estratégias funcionais e com os processos de negócio, para que os processos sejam desenhados de forma a auxiliar na realização da missão do negócio.

O patrocínio para a realização da mudança é fundamental e deve ser exercido pela alta administração da organização, pois demonstra para o grupo de trabalhadores a intenção de evoluir, de desenvolver o negócio. Esta declaração de intenções também é evidenciada através do estabelecimento de metas claras, que orientem os processos e as pessoas para que auxiliem na execução da estratégia corporativa e funcional. 
Os fatores que complementam os pontos de atenção que devem ser observados na implantação do BPM são o estabelecimento de um dono para o processo, que possa ser responsável e responsabilizado pela execução das atividades do processo e o estabelecimento de métricas que permitam controlar o processo e monitorar seu alinhamento com os objetivos estratégicos do negócio. Já as estratégias para implementação do ciclo de vida do BPM podem ser orientadas por um conjunto de técnicas, métodos e conhecimentos aplicados no gerenciamento de projetos, que é definido como sendo um esforço temporário para desenvolver um produto ou serviço com características únicas (PMBOK, 2008). Os aspectos que serão considerados neste projeto foram escopo, tempo, qualidade, recursos humanos, ações de comunicações e riscos. Os aspectos relativos a custos e aquisição, que completam o conjunto de conhecimento abordados pelo gerenciamento de projetos, não fizeram parte deste estudo.

O ciclo do gerenciamento de projetos pode ser sumarizado da seguinte forma (PMI, 2008): (i) Iniciação: define e autoriza o projeto ou uma fase do projeto; (ii) Planejamento: define e refina os objetivos e planeja a ação necessária para alcançar os objetivos e o escopo para os quais o projeto foi realizado; (iii) Execução: integra pessoas e outros recursos para realizar o plano de gerenciamento do projeto para o projeto; (iv) Monitoramento e Controle: mede e monitora regularmente o progresso para identificar variações em relação ao plano de gerenciamento do projeto, de forma que possam ser tomadas ações corretivas quando necessário para atender aos objetivos do projeto; e (v) Encerramento: formaliza a aceitação do produto, serviço ou resultado e conduz o projeto ou uma fase do projeto a um final ordenado.

O objetivo de considerar o uso de um método para a gestão do projeto de implementação de BPM durante sua fase inicial, é aumentar o controle no desenvolvimento das ações para que atendam aos requisitos definidos no escopo do projeto e possibilitem a execução ordenada das tarefas necessárias. Não é a finalidade deste estudo associar os conceitos de gestão de projetos com os de gestão de processos de negócio, mas somente gerenciar o projeto de implementação do ciclo de gestão de processos.

Outro aspecto que deve ser considerado no projeto de implementação da prática de gestão do BPM, principalmente na fase de implementação (transformação), é a forma como a mudança ou transformação será conduzida e gerenciada. O modelo proposto é baseado no ciclo de gestão da mudança criado por Kotter (1997), que é dividido em oito etapas: (i) Demonstre a urgência: ajude os outros a ver a necessidade de mudança e a importância de uma ação imediata; (ii) Reúna a equipe orientadora: certifique-se de que um grupo seguro orientará a mudança, um grupo com habilidades de análise e de liderança, credibilidade, capacidade de comunicação e consciência da urgência; (iii) Desenvolva a visão da mudança e a estratégia adequada: esclareça como o futuro será diferente do passado e como é possível torná-lo realidade; (iv) Comunique-se para ser 
entendido e apoiado: faça com que o maior número possível de pessoas entenda e aceite a estratégia; (v) Divida responsabilidades: remova o máximo possível de obstáculos, facilitando a ajuda de todos que querem tornar realidade a mudança; (vi) Demonstre vitórias de curto prazo: divulgue os sucessos sempre que eles aconteçam, sejam grandes ou pequenos; (vii) Não relaxe: pressione cada vez mais após os primeiros sucessos; e (viii) Crie uma nova cultura: reforce os novos comportamentos e certifique-se de que serão bem-sucedidos até se tornarem fortes para substituir as antigas tradições.

Após estas considerações, é possível definir as estratégias a serem utilizadas em cada fase da implementação do ciclo de vida BPM proposto pela ABPMP. Destaca-se que o foco deste estudo não é esgotar o tema pesquisado, mas sim criar um roteiro factível para a execução deste projeto, que considere fortemente a estratégia do negócio, a cultura da organização pesquisada e a competência dos seus recursos humanos.

\subsection{Planejamento e estratégia}

A primeira fase do ciclo proposto tem elevada importância, pois é o momento de direcionamento das ações que serão tomadas para alinhar as pessoas, processos, estratégia e sistemas. Este direcionamento obrigatoriamente passa pela definição do escopo do projeto, quais os objetivos devem ser alcançados e quais recursos serão utilizados para produzir o resultado esperado.

Como estratégia para definição do plano para a implementação do ciclo de vida propõe-se a realização de um fórum com todos stakeholders para conscientização da necessidade de mudança e para identificação das restrições e expectativas dos envolvidos com relação ao processo. É essencial garantir o patrocínio e apoio incondicional de um executivo da alta administração.

Atualmente, a organização possui uma visão totalmente departamental, onde cada área do negócio é responsável pela execução de suas atividades rotineiras e também é avaliada por indicadores que medem esta eficiência setorial. Sua estrutura hierárquica segue o modelo matricial tradicional, concentrando e especializando o conhecimento das pessoas em seus cargos, aumentando a importância na medida em que sobe na estrutura organizacional.

Sendo assim, propõe-se a realização de um teste piloto no processo de gestão do ciclo do pedido da organização, por este apresentar uma peculiaridade na sua execução, oriunda da estratégia de produção adotada para atender a demanda. A partir disso, será necessário replicar a aplicação dos métodos de gestão de processos em toda organização, gerando ganhos em todas as atividades desenvolvidas, sejam elas primárias, de suporte ou gerenciamento.

\subsection{Análise de pocesso}


Nesta fase do projeto, deve-se criar um entendimento comum entre todos os participantes da real situação do processo analisado, independente dos acertos ou erros encontrados. Deve-se verificar seu alinhamento com a estratégia, sua capacidade de entregar seus produtos dentro dos requisitos acordados e a eficiência com que utiliza os recursos durante a execução das atividades.

A análise proposta é baseada em entrevistas individuais com os principais participantes do processo e também com os grupos de interesse envolvidos (clientes e parceiros de negócio). Após as entrevistas individuais, propõe-se um novo fórum com todos os participantes para que compreendam todas as atividades envolvidas no atendimento ao cliente e nivele-se o conhecimento do negócio entre os participantes.

Esta análise deve proporcionar uma visão holística, de todos as etapas necessárias para o atendimento das necessidades do cliente, demonstrando a situação atual do ciclo do pedido na empresa estudada. Nesta análise se utilizará a técnica de documentação SIPOC (supplier, input, process, output and customer), que consiste em mapear todos os fornecedores, os insumos, as atividades do processamento, os produtos gerados e os clientes para cada uma das atividades contidas nos quatro processos propostos. Em complemento à técnica do SIPOC, devem ser mapeadas as regras de negócio envolvidas em cada atividade, as métricas utilizadas para medir a eficiência e eficácia do processo e os recursos envolvidos (financeiros, humanos e tecnológicos).

Como resultado desta fase de investigação, deve-se gerar uma lista com diversas oportunidades de melhoria que serão levantadas ao longo da análise. Estas oportunidades devem refletir as ações para a eliminação das causas dos problemas encontrados, e não dos efeitos. Estas oportunidades ainda não respondem o "como" resolver os problemas encontrados, mas sim "o que" deve ser melhorado. O caminho que deve ser seguido será tratado na fase de desenho.

As oportunidades de melhoria representam pontos com problemas ou falhas no processo, ou com potencial relevante para o erro. Estes pontos podem ser classificados quanto a sua natureza: reais ou potenciais falhas no modo de fazer as coisas (métodos), na utilização ou falta de recursos disponíveis (ferramentas computacionais ou não) ou nas competências das pessoas que utilizam as ferramentas e métodos (conhecimentos, habilidades e atitudes).

\subsection{Desenho e modelagem de processo}

A partir das oportunidades de melhoria identificadas na fase anterior, deve-se desenhar a estrutura que o processo terá no futuro. Esta estrutura envolve definições a do dono do processo, dos roteiros, regras, papéis, métodos e métricas que serão utilizadas para que o processo atinja seu objetivo. A eliminação de qualquer atividade que não agrega valor ao negócio será umas das premissas deste novo desenho. 
Como forma de estruturação do processo, propõe-se uma visão ponta a ponta do processo, fazendo um corte horizontal nos departamentos da organização e agrupando todas as atividades envolvidas no ciclo de atendimento ao pedido do cliente, reunindo-as nos seguintes processos: planejar as vendas, processar os pedidos, expedir e prestar serviços pós-vendas, o que será denominado "atender mercado", conforme demonstrado na Figura 1:

Figura 1 - Estrutura do processo "atender mercado"

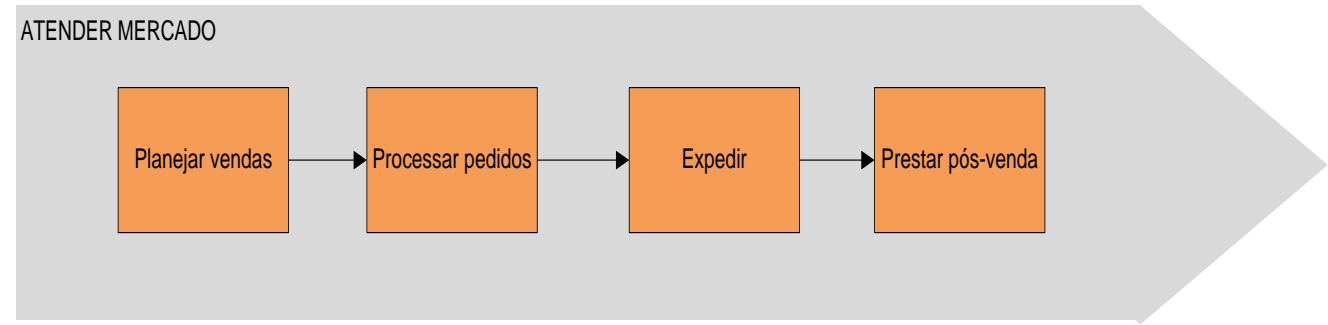

Fonte: Elaborada pelos autores.

Como o objetivo deste processo redesenhado é aumentar a eficiência no atendimento ao cliente, deve-se eliminar as filas e esperas a que o processo está submetido nos departamentos, assegurando um fluxo contínuo e reduzindo o ciclo de tempo para realização das atividades. Uma das formas de reduzir estas filas departamentais (tempos de espera) é eliminar a visão por funções e iniciar a gerir as atividades orientadas aos processos, medindo a contribuição de cada atividade para a entrega de valor ao cliente.

Esta visão do ciclo completo de atendimento ao cliente (incluindo áreas tipicamente associadas à área industrial, como expedição pós-vendas) é baseada no modelo de referência PCF (Process Classification Framework) desenvolvido pela APQC - American Productivity and Quality Council (APQC, 2010) e foi adotado como o benchmarking para o processo. Posteriormente, o desdobramento e detalhamento dos processos em atividades deverão ser realizados observando o mesmo modelo de referência.

\subsection{Implementação de processo}

Neste momento todas as oportunidades de melhoria e proposições sobre os processos melhorados ou redesenhados já devem ter sido exaustivamente discutidas. O objetivo desta fase é criar um plano factível para conduzir o processo do estado atual para o estado desejado, tornado o seu desenho operacional. É fundamental neste momento a adequação das competências dos recursos humanos da organização com as competências necessárias para a execução do novo processo.

O foco nesta fase é garantir que os recursos humanos da organização aceitem a mudança da melhor forma possível, tornando mais rápido a incorporação das novas definições de regras de negócio, procedimentos e métricas. Um fator determinante para realização da mudança é o patrocínio da alta administração, pois entende-se que a aceitação e apoio dos executivos transmite 
para a organização a motivação e incentivo necessário para atravessar com sucesso o período de mudança.

A criação do plano de ações para execução das mudanças propostas será o instrumento adotado para conduzir as atividades neste período. O plano de ação será formatado utilizando-se o detalhamento 5W2H (what, why, where, when, who, how, how much - o que, por que, onde, quando, quem, como, quanto custa), e será conduzido com base no ciclo de gerenciamento de projetos proposto pelo PMI (2008), pois o conjunto de ações que será gerado nesta fase se caracteriza como projetos, e como tal devem ser gerenciado.

\subsection{Monitoramento e medição de processo}

A fase de monitoramento e medição funciona como o termômetro do processo, medindo constantemente os resultados entregues pelo processo e comparando-os aos objetivos previamente estabelecidos. Este constante monitoramento permite realizar os ajustes necessários, no momento adequado e ao menor custo possível, proporcionando a correção imediata aos possíveis desvios ou também a adoção de oportunidades de melhoria que surgem no curso da ação.

A coleta e o acúmulo de informações relevantes ao longo da execução dos processos passa pelo estabelecimento de métricas que possam avaliar tanto o resultado (eficácia) quanto o desenvolvimento das atividades (eficiência) estabelecendo-se como um instrumento essencial para suportar a tomada de decisão dos gestores.

A construção de uma métrica que possa monitorar o alcance dos objetivos do processo e sua contribuição para a realização da estratégia empresarial passa obrigatoriamente pelo estabelecimento de indicadores relevantes, que alinhem o processo com os objetivos estratégicos, permitam predizer as tendências das atividades que estão sendo monitoradas, que sirvam como estímulo e meta para os participantes do processo e que qualifiquem a tomada de decisão do gestor, com informações precisas e pertinentes.

\subsection{Refinamento de processo}

O refinamento consiste na adoção de ações emergentes ou deliberadas para aperfeiçoar o desempenho dos processos, melhorando-os continuamente até que seja necessário inovar, quebrar paradigmas, repensar as atividades que estão sendo realizadas. A constante evolução dos processos gera um importante aprendizado para a organização, e este conhecimento deve ser registrado, tornando-se um repositório de soluções para ser utilizado oportunamente no futuro.

Esta última fase do ciclo de vida BPM proposto pela ABPMP funciona como a realimentação de todo o ciclo, gerando informações e subsídios para que constantemente se evolua do estado atual para o desejado em um movimento contínuo e incessante, evoluindo e adaptando-se 
as necessidades do cliente para buscar a perpetuação da organização, pois uma organização que aprende e evolui constantemente desenvolve como competência principal a flexibilidade que o mercado exige dos competidores atuais.

\subsection{Mais alguns comentários sobre Tecnologia da Informação (TI)}

O desenvolvimento das tarefas necessárias para a execução de todas as etapas do ciclo de vida de gestão de processos na organização dependerão fortemente do uso de sistemas computacionais que habilitem e auxiliem estas tarefas. A utilização de uma suíte BPM será essencial para permitir a utilização das metodologias e estratégias propostas, na medida em que o volume de informações e a complexidade do processo não permitem seu controle manual.

O BPM a ser escolhido deverá conter as seguintes capacidades para atender as necessidades da organização: (i) captar, identificar e modelar os processos selecionados; (ii) operar o esquema de identificação, o sequenciamento e a interação desses processos; (iii) tornar possível a integração do sistema de gestão de processos com o ambiente de TI; (iv) aceitar o conjunto de critérios e métodos (metodologia) adotados no projeto; (v) fornecer e colocar disponível, a tempo e na hora certa, informações sobre esses processos; (vi) possibilitar o monitoramento de atividades, o funcionamento e desempenho dos processos; (vii) fornecer ferramentas para simulação e otimização dos processos; e (viii) fornecer recursos e facilidades para a implementação de ações, visando à obtenção de resultados planejados e à melhoria contínua desses processos.

\subsection{Roteiro para implementação do ciclo de vida BPM}

O roteiro proposto para implementação do BPM na organização é baseado no ciclo de vida da gestão de processos propostos pela ABPMP (2009), sendo estruturado também para atender as fases do ciclo do gerenciamento de projetos do PMI (2008). Neste sentido, entende-se como elemento crítico para o sucesso deste projeto de implementação a adoção de um método para gerenciar a mudança resultante da adoção desta técnica de gestão. Por isso, o roteiro proposto contempla as etapas do ciclo de gestão da mudança proposto por Kotter (1997). A matriz a seguir contém as fases propostas pelos três ciclos de gestão de processos, projetos e mudanças (Figura 2).

Figura 2 - Matriz de comparação entre os ciclos

\begin{tabular}{|l|llllll|}
\hline \multicolumn{1}{|l}{ Ciclo } & \multicolumn{2}{c}{ PMI (2008) } & \multicolumn{1}{c|}{ Kotter (1997) } & ABPMP (2009) \\
\hline \multirow{3}{*}{ Fases } & 1 & Iniciar & 1 & $\begin{array}{l}\text { Demonstrar urgência } \\
\text { Reunir equipe orientadora }\end{array}$ & 1 & \\
\cline { 2 - 7 } & 2 & Planejar & 2 & $\begin{array}{l}\text { Desenvolver visão da mudança } \\
\text { e a estratégia }\end{array}$ & 2 & Planejar
\end{tabular}




\begin{tabular}{|l|llllll|} 
& \multicolumn{3}{|l|}{ Comunicar } & \\
\cline { 2 - 6 } & Executar & 3 & $\begin{array}{l}\text { Dividir responsabilidades } \\
\text { Demonstrar vitórias }\end{array}$ & 3 & $\begin{array}{l}\text { Analisar } \\
\text { Desenhar e Modelar } \\
\text { Implementar }\end{array}$ \\
\hline & & Monitorar e Controlar & 4 & Não relaxar & 4 & Monitorar e Medir \\
\hline 5 & & 5 & Criar uma nova cultura & 5 & Refinar \\
\hline 6 & Encerrar & 6 & & 6 & \\
\hline
\end{tabular}

Fonte: Elaborada pelos autores.

Como resultado da compilação destes conceitos e dos aspectos relativos à empresa pesquisada, propõe-se uma estratégia baseada no roteiro demonstrado na Figura 3.

Figura 3 - Roteiro proposto para implementação do ciclo de vida BPM

\begin{tabular}{|c|c|c|}
\hline Fases & Etapas & Descrição \\
\hline \multicolumn{3}{|c|}{1 - Planejar } \\
\hline 1.1 & Definir escopo do projeto & etapa de definição de requisitos, objetivos e recursos para o projeto \\
\hline 1.2 & Definir estratégia & etapa de determinação da estratégia de comunicação e ação \\
\hline 1.3 & Realizar fórum de abertura & etapa de comunicação e conscientização da necessidade de mudança \\
\hline \multicolumn{3}{|c|}{2 - Analisar } \\
\hline 2.1 & Realizar entrevistas & etapa de coleta de dados e pesquisa \\
\hline 2.2 & $\begin{array}{l}\text { Realizar fórum de nivelamento de } \\
\text { conhecimentos }\end{array}$ & etapa de comunicação para compreensão geral do processo \\
\hline 2.3 & Criar Documentação SIPOC & etapa de documentação e estruturação do processo \\
\hline 2.4 & Apontar oportunidades de melhoria & etapa de divulgação das oportunidades de melhorias levantadas na análise \\
\hline \multicolumn{3}{|c|}{3 - Desenhar e Modelar } \\
\hline 3.1 & Desenhar estrutura do processo & etapa de desenho física das atividades necessárias a execução do processo \\
\hline 3.2 & Realizar benchmark & etapa de comparação e adequação da realidade ao modelo de referência \\
\hline 3.3 & Detalhar estrutura do processo & etapa de desdobramento das atividades do processo em tarefas \\
\hline \multicolumn{3}{|c|}{4 - Implementar } \\
\hline 4.1 & Garantir patrocínio para a mudança & etapa de comunicação e realinhamento do escopo do projeto e da estratégia \\
\hline 4.2 & Treinar e capacitar & etapa de preparação dos recursos humanos para as adequações propostas \\
\hline 4.3 & Criar plano de ação $5 \mathrm{~W} 2 \mathrm{H}$ & etapa de definição dos prazos, meios e recursos necessários para realizar a mudança \\
\hline 4.4 & Executar & etapa de aplicação das definições geradas \\
\hline \multicolumn{3}{|c|}{5 - Monitorar e Medir } \\
\hline 5.1 & Estabelecer métricas & etapa de definição das formas de controle aplicadas ao processo \\
\hline 5.2 & Coletar dados & etapa de coleta e acúmulo de informações para a tomada de decisão \\
\hline \multicolumn{3}{|c|}{6 -Refinar } \\
\hline 6.1 & Evoluir & etapa de realização de ajustes e correções \\
\hline
\end{tabular}

Fonte: Elaborada pelos autores.

\section{Resultados esperados}

A adoção do BPM como prática de gestão na busca da melhoria do atendimento ao cliente deve proporcionar um aumento no desempenho da organização pesquisada, uma vez que entre as premissas propostas para a melhoria do processo está a eliminação de qualquer atividade que não agregue valor ao negócio. Esta busca pelos desperdícios existentes na organização resultará em ganhos de tempo e custo, aumentando o nível de serviço oferecido ao cliente.

A metodologia de gestão de processos propostas pela ABPMP mostra-se estruturada de forma lógica e ordenada temporalmente, contemplando os aspectos necessários a realização de uma proposta desta magnitude. A divisão das atividades em grandes fases parece adequada para criar o envolvimento e entendimento gradual da equipe participante do projeto, facilitando a busca das soluções e sua respectiva implementação. 
As estratégias propostas para a implementação do ciclo de vida de gestão de processos contemplam também aspectos dos ciclos de gestão de projetos e gestão de mudanças, como forma de contribuir com tópicos específicos e complementares ao tema. A implementação tem características de um projeto, como: escopo, recursos humanos, prazos e produtos planejados previamente, o que torna o uso do ciclo de gestão de projetos necessário. Outro aspecto relevante na implementação é o uso das técnicas de gestão da mudança para conduzir de maneira apropriada este período de descobrimento, reflexão, projeção e adoção de novos métodos e práticas, que modificará a rotina das pessoas.

A orientação do trabalho por um roteiro definido deve facilitar a condução do processo de mudança e proporcionar controle sobre os aspectos de prazo e alocação de recursos, garantindo que os objetivos de cada fase sejam alcançados com a qualidade necessária. O gerenciamento deste período de implementação permitirá que o planejamento seja executado de maneira adequada.

As características definidas como necessárias para a adoção de uma ferramenta computacional para habilitar a gestão de processos na organização é compatível com as soluções encontradas no mercado atualmente, não representando dificuldade para sua adoção. As características específicas de cada ferramenta disponível no mercado não são objetivo deste estudo, mas representam um campo de estudo futuro a ser considerado em uma nova proposta.

Um aspecto bastante relevante na realização deste projeto é a forma de comunicação que será adotada ao longo de todas as fases propostas. A escolha da estratégia de comunicação deverá contemplar a efetiva transmissão e compreensão das intenções do trabalho, esclarecendo para todos trabalhadores o objetivo do projeto. A transparência desta comunicação durante todo o período facilitará a aceitação das mudanças propostas e diminuirá a chance de boicotes. Deve-se utilizar todos os recursos disponíveis, como intranet, murais, jornal interno e demais ferramentas para garantir a mobilização da força de trabalho neste período.

A apresentação da situação atual do ciclo do pedido na organização, que é um dos objetivos secundários deste projeto, somente será possível após a fase de análise e desenho dos processos, proporcionando assim descobrir, com base em fatos e dados, a real condição deste processo de atendimento ao cliente. A primeira etapa da metodologia proposta é o levantamento e investigação dos aspectos relacionados ao processo, e entende-se que antes da realização desta fase não é possível concluir um diagnóstico preciso.

Outro aspecto relevante que impactará na investigação proposta é a estratégia de produção adotada pela empresa (estratégia ETO). Esta estratégia de produção tem como característica um ciclo de pedido mais longo, devido ao fato de serem necessárias várias rodadas de ajustes entre a necessidade do cliente, o portfólio de produtos oferecidos e o custo da solução encontrada. 
No macroprocesso proposto, denominado "Atender Mercado", os processos estão subdivididos em planejar as vendas, processar os pedidos, expedir e prestar serviços pós-vendas. O processo mais afetado pela escolha desta estratégia de produção é o de processamento de pedidos, pelas razões já apresentadas anteriormente. Este processo deverá ser priorizado no momento da geração de soluções pois é o processo que tem o maior número de recursos alocados, e que possivelmente representa uma restrição a continuidade do fluxo de todo o macro-processo.

Um aspecto abordado pela disciplina de gerenciamento de projetos apresentada anteriormente e que não tem aplicação na proposta de implementação da gestão de processos é a fase de encerramento do projeto. A gestão de processos se caracteriza pela continua e incessante evolução de todas as atividades envolvidas na realização de um processo, não apresentando portanto um final ou término.

Os resultados a serem alcançados com a adoção da prática de gestão BPM serão possíveis de ser determinados somente após a realização do desenho do estado futuro do processo, após cumprida as etapas de planejamento, análise e modelagem, para que sejam identificadas de maneira apropriada os pontos do processo que devem ser melhorados e, desta forma poder determinar o real potencial de ganho nos processos oriundos da implementação do estado desejado para o processo. As mudanças decorrentes do uso de novas tecnologias de informação dependerão da ferramenta adotada, não permitindo uma projeção imediata, limitando neste aspecto o resultado da pesquisa.

Os riscos associados a implementação desta técnica de gestão e suas ferramentas estão associados principalmente a definição clara dos objetivos da organização em relação a mudança, a ausência de uma cultura voltada a execução e o risco de não aceitação da mudança, o apego ao status quo e ao modus operandi. Os maiores riscos a que o projeto está submetido são os relacionados à gestão aos recursos humanos dentro do processo de mudança e devem ser minimizados conforme estratégia citada anteriormente.

\section{Considerações finais}

A adoção do BPM proposto pela ABPMP como prática de gestão em uma organização aumenta fortemente a chance de sucesso desta organização no cenário competitivo atual, porque atua como uma forma de estruturação das atividades desenvolvidas na empresa com o objetivo de satisfazer necessidades e expectativas do cliente. Esta forma de atuação, de atender as necessidades dos clientes, parece óbvia para uma organização comercial, mas nem sempre é praticada, e a prova disso são os números crescentes de reclamações junto aos órgãos de defesa dos direitos do consumidor.

O encadeamento desta prática de gestão em um ciclo de vida permite a organização praticante evoluir constantemente com seus processos, uma vez que este conjunto de fases ou etapas 
é cíclico e permite elevar o nível de maturidade dos processos ao longo do tempo, incutindo na empresa uma necessidade de inovar constantemente. Esta inovação permite a empresa realizar de forma diferente sua entrega de valor ao cliente, garantindo uma vantagem competitiva sustentável.

A estruturação de um roteiro para implementação do ciclo de vida BPM apoiado por outras técnicas de gestão, como de projetos e de mudanças, assim como o uso de modelos de referência, torna o caminho para organização mais previsível, diminuindo a incerteza ao longo do projeto. Esta estrutura de projeto, segmentada em fases, com sua distribuição de tarefas e responsabilidades, permite que o processo de compreensão seja lúdico, possibilitando o encontro de soluções para os problemas identificados de forma eficaz.

A impossibilidade de prever resultados específicos para o projeto antecipadamente não diminui a relevância das técnicas de gestão e de suas ferramentas de tecnologia, somente reforça o conceito do ciclo de vida da gestão de processos. Também será possível determinar de maneira precisa a real situação da organização apenas após a execução de um diagnóstico nas fases de análise, desenho e modelagem.

A conexão entre a estratégia de atendimento à demanda adotada pela empresa e o BPM está vinculada ao fato de que as atividades relacionadas ao processamento de pedido, um dos processos propostos para o macro-processo atender mercado, serem complexas e onerosas, e portando, a definição do conjunto de atividades que serão realizadas devem considerar de maneira racional como priorizar este objetivo de desempenho da organização e ao mesmo tempo aumentar a eficiência operacional do processo.

O caráter científico e exploratório dos métodos adotados na gestão de processos proposto pela ABPMP durante todo o ciclo de vida visam aumentar significativamente a possibilidade de sucesso na organização praticante e reforçam a necessidade de evoluir constantemente, buscando alternativas eficientes e eficazes para oferecer aos clientes uma proposta de valor genuína e duradoura. Acredita-se que a construção da vantagem competitiva sustentável passa pela elaboração de processos que tenham como objetivo principal tornar o relacionamento do cliente com a organização mais fácil, rápido, barato, personalizado e prazeroso, assim como melhorar continuamente sua eficiência, permitindo a construção de uma relação única, difícil de ser imitada pelos concorrentes e que proporciona a organização condições de evoluir e perpetuar-se.

\footnotetext{
Abstract

The article presents a process management methodology based on management process lifecycle proposed by ABPMP - Association of Business Process Management Professionals as well as activities, techniques and management practices that enable the realization of BPM - Business Process Management, including the planning and strategy, process analysis, design and process modeling, process implementation, monitoring a process control and refinement of process phases. As a complement, were presented the tools that enable and support the lifecycle management
} 
processes. So, we propose the use of a roadmap for applying the methodology in a metallurgical company to increase the efficiency of its orders cycle. With this proposed intervention is expected to have a greater connection between the demand attending strategy adopted by the company (ETO - Engineer-to-Order) and its productive process managing, resulting in better customer service.

Key-words: BPM - Business Process Management; process management; production strategy.

\section{Referências}

ABPMP - Association of Business Process Management Professionals. BPM CBOK - Business Process Management: common body of knowledge. Chicago: ABPMP, 2009.

APQC - American Productivity and Quality Council. PCF - Process Classification Framework. Disponível em: www.apqc.org. Acessado em: 10 set. 2010.

ANDRADE, M. M. Introdução à metodologia do trabalho científico: elaboração de trabalhos na graduação. 7. ed. São Paulo: Atlas, 2006.

BALASUBRAMANIAM, R.; RADHIKA, J.; NISSEN, M.; XU, P. Managing context in business process management systems. Requirements Engineering, v. 10, n. 3, p. 223-237, 2005.

cross

BALDAM, R.; VALLE, R.; PEREIRA, H. Gerenciamento de processos de negócios: Business Process Manager. 2. ed. São Paulo: Érica, 2008.

CHENG, C. S. Concurrent engineering-to-order operation in the manufacturing engineering contracting in industries. International Journal of Industrial and Systems Engineering, v. 1, n. 1, p. 37-58, 2006.

cross ref

CORRÊA, H. L.; CORRÊA, C. A. Administração da produção e operações: manufatura e serviços: uma abordagem estratégica. 2. ed, São Paulo: Atlas, 2006.

CORRÊA, H. L.; GIANESI, I. G. N.; CAON, M. Planejamento, programação e controle da produção: MRP II/ERP - conceitos, uso e implantação-base para SAP, Oracle applications e outros softwares integrados de gestão. 5. ed. São Paulo: Atlas, 2008.

CRUZ, T. BPM \& BPMS. Rio de Janeiro: Brasport, 2008.

DAVENPORT, T. H. Reengenharia de processos: como inovar na empresa através da tecnologia de informação. Rio de Janeiro: Campus, 1994.

DAVIS, M. M.; AQUILANO, N. J.; CHASE, R. B. Fundamentos da administração da produção. 3. ed. Porto Alegre: Bookman Editora, 2001.

DE SORDI, J. O. Gestão por processos: uma abordagem da moderna administração. São Paulo: Saraiva, 2005.

FNQ - Fundação Prêmio Nacional da Qualidade. Conceitos fundamentais da excelência em gestão. Disponível em: www.fnq.org.br. Acessado em: 22 abr. 2010.

FORTIN, C.; HUET, G. Manufacturing process management: iterative synchronization of engineering data with manufacturing realities. International Journal of Product Development, v. 4, n. 3/4, p. 280-295, 2007.

cross ref

GIL, A. C. Métodos e técnicas de pesquisa social. São Paulo: Atlas, 1999.

GONÇALVES, J. E. L. As empresas são grandes coleções de processos. RAE - Revista de Administração de Empresas, v. 40, n. 1, p. 6-19, jan-mar. 2000a.

GONÇALVES, J. E. L. Processo, que processo? RAE - Revista de Administração de Empresas, v. 40, n. 4, p. 1-19, out./dez. 2000 b. 
GODOY, A. S. Introdução à pesquisa qualitativa e suas possibilidades. Revista de Administração de Empresas, v. 35, n. 2, p. 57-63, mar./abr, 1995.

HAMMER, M. A agenda: o que as empresas devem fazer para dominar esta década. Rio de Janeiro: Campus, 2001.

HAMMER, M.; CHAMPY, J. Reengenharia: repensando a empresa em função dos clientes, da concorrência e das grandes mudanças da gerência. Rio de Janeiro: Campus, 1994.

HARRINGTON, H. J. Business process improvement: the breakthrough strategy for total quality, productivity, and competitiveness. New York: McGraw-Hill, 1991.

HENNINK, M.; HUTTER, I.; BAILEY, A. Qualitative research methods. Thousand Oaks: Sage Publications, 2011.

HUANG, X. X.; MEI, S. Z. Allying business process reengineering with strategy: a new perspective for BPR. International Journal of Internet and Enterprise Management, v. 1, n. 3, p. 281-292, 2003.

cross ref

HICKS, C.; MCGOVERN, T. Product life cycle management in engineer-to-order industries. International Journal of Technology Management, v. 48, n. 2, p. 153-167, 2009.

cross ref

JESTON, J. High performance management. Industrial Engineer, v. 40, n. 5, p. 33-37, 2008.

JESTON, J.; NELIS, J. Business process management: practical guidelines to successful implementations. Oxford: Elsevier, 2006.

KLIPPEL, M.; ANTUNES, J. A. V., Jr.; PELLEGRIN, I. Os circuitos de planejamento, programação e controle da produção e dos materiais para indústrias do tipo engineer-to-order - pedidos contra projetos de engenharia. In: XXV ENEGEP - Encontro Nacional de Engenharia de Produção. Anais... Porto Alegre, 2005.

KOTTER, J. P. Liderando mudanças. 9. ed. Rio de Janeiro: Campus, 1997.

MANOILOV, G.; DELIISKA, B. Ontological model of business process management systems. In: AIP Conference. Proceedings... p. 491-499, 2008.

NEAL, K. Driving better business performance with document management processes. The Information Management Journal, v. 42, n. 6, p. 48-50, 2008.

MARCONI, M. A.; LAKATOS, E. M. Técnicas de pesquisa. 6. ed. São Paulo: Atlas, 2007.

OLIVEIRA, D. P. R. Administração de processos: conceitos, metodologias, práticas. 3. ed. São Paulo: Atlas, 2009.

PAIM, R.; CARDOSO, V.; CAULLIRAUX, H. Gestão de processos: pensar, agir e aprender. Porto Alegre: Bookman, 2009.

PMI - Project Management Institute. PMBOK - Project Management: common body of knowledge. $4^{\text {th }}$ edition. Pennsylvania: PMI, 2008.

PORTER, M. E. Estratégia competitiva: técnicas para a análise de indústrias e da concorrência. 30. ed. Rio de Janeiro: Campus, 2005.

ROSEMANN, M.; RECKER, J.; FLENDER, C. Contextualisation of business process. International Journal of Business Process Integration and Management, v. 3, n. 1, p. 47-60, 2008.

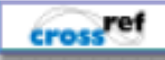

RUMMLER, G. A., BRACHE, A. P. Melhores desempenhos das empresas. 2. ed. São Paulo: Makron Books, 1994.

SILVA, E. L.; MENEZES, E. M. Metodologia da pesquisa e elaboração de dissertação. 3. ed. Florianópolis: Laboratório de Ensino à Distância da UFSC, 2001.

SLACK, N. et al. Administração da produção. São Paulo: Atlas, 1999. 
SLACK, N.; LEWIS, M. Towards a definitional model of business process technology. International Business of Process Management and Benchmarking, v. 1, n. 1, p. 3-24, 2005.

\section{cross ref}

SMITH, H.; FINGAR, P. Business Process Management - the third wave. Tampa: Meghan Kiffer, 2003.

TRACY, R. P. IT security management and business process automation: challenges, approaches, and rewards. Information Systems Security, v, 16, p. 114-122, 2007.

TRAPPEY, C. V.; TRAPPEY, A. J. C.; CHIANG, T. A.; KUO, J. Y. A strategic product portfolio management methodology considering $R \& D$ resource constraints for engineering-to-order industries. International Journal of Technology Management, v. 48, n. 2, p. 258-276, 2009.

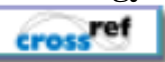

REMLER, D. K.; VAN RYZIN, G. G. Research methods in practice: strategies for description and causation. Thousand Oaks: Sage Publications, 2011.

WHEELWRIGTH, S. Manufacturing strategy: defining the missing link. Strategic Management Journal, v. 5, n. 1, p. 77-91, 1984.

cross $r$

WOLTER, C.; MEINEL, C. Na approach to capture authorization requirements in business process. Requirements Engineering, v. 15, n. 4, p. 359-373, 2010.

\section{cross ref}

\section{Dados dos autores:}

\section{Nome: Gabriel Sperandio Milan}

Filiação institucional: Universidade de Caxias do Sul (UCS)

Centro: Centro de Ciências da Administração

Função: Professor pesquisador ligado aos programas de Mestrado e Doutorado em Administração Endereço para correspondência: Rua: Francisco Getúlio Vargas, 1130 - PPGA / Bloco F - 4 andar

- Bairro: Jardim América - 95070-560 - Caxias do Sul - RS

Telefones para contato: (54) 3218.2100

e-mail:gsmilan@ucs.br

\section{Nome: Francis André Soso}

Filiação institucional: Universidade de Caxias do Sul (UCS)

Função: Pesquisador e Especialista em Gestão Estratégica da Qualidade

Endereço para correspondência: Rua: Francisco Getúlio Vargas, 1130 - PPGA / Bloco F - 4º andar

- Bairro: Jardim América - 95070-560 - Caxias do Sul - RS

Telefones para contato: (54) 3218.2100

e-mail: soso.francis@gmail.com

Enviado em: 07/02/2011

Aprovado em: $15 / 06 / 2012$ 